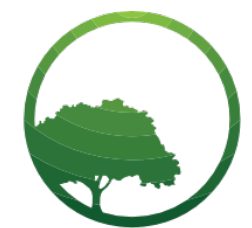

Business \& Social Science IJRBS

\section{Research in Business and Social Science}

IJRBS Vol 6 No 3, ISSN: 2147-4478

Contents available at www.ssbfnet.com/ojs http://dx.doi.org/10.20525/ijrbs.v6i3.750

\title{
Administrative and managerial patterns on the RtHC in the Makhado municipality rural clinics
}

\section{Tshimangadzo Selina Mudau}

Department of Nursing Science, Sefako Makgatho Health Sciences University, GaRankuwa, Republic of South Africa (+27 82573 9589)

\section{Sarie P. Human}

Department of Health Studies, University of South Africa, Pretoria, Republic of South Africa (Tel: +27 12429 6290)

\begin{abstract}
This paper investigates the administrative and managerial pattern on the Road to Health Chart (RtHC) among clinics in the Makhado municipality. Retrospective observations of the processes of recordings the nurses performed on the RtHC $(n=36)$ were carried out to collect data. A checklist was used to aid the observations. Furthermore, face-to-face interviews were administered on participant nurses in collaboration with document review. The results of this paper revealed considerable omissions on the administration and management of the RtHC instrument. Crucial information was also incorrectly recorded while some was never recorded at all. Clinic managers should continuously monitor the nurses on the administration and management of the RtHC to ensure quality.
\end{abstract}

Key words: Child health, Growth Monitoring, Registered Nurses, Road to Health Chart (RtHC) JEL classification: C51, M15, M31 


\section{Introduction}

This paper is built on premise that evidence exists that the South African health system is characterised by serious omissions with regard the administration and management of the RtHC by public health workers, and in particular in state service facilities (Senoge, 2011; Mulaudzi, 2012; Win, 2016). Senoge (2011) found that level of knowledge and performance in the administration and management of the RtHC is unacceptably lower than expected considering that approximately $62 \%$ of public health workers struggled with the technical issues of the administration and mnagement of the RtHC instrument. This paper adopted its theoretical underpinnings from Win (2016) - especially with regard analysis of the complexities, barriers and bottlenecks affecting proper administration and management of the RtHC among the selected participant clinics. The variables guiding this paper were constructed from related studies (Senoge, 2011; Win, 2016) and departmental guidelines on the RtHC.

This is a quantitative-qualitative study. This section describes the procedure followed in conducting the study; that is the ethical considerations of the study, the nature of the study, the sampling method and procedures, the method and instruments used in the collection, analysis and interpretation of data, and finally the theoretical underpinnings informing the analysis of the study. The results of this paper are thereafter reported, conclusions and policy recommendaions presented.

Ethical clearance was granted by the University of South Africa (UNISA) where the main study was academically registered and conducted from. The Limpopo Provincial Department of Health also sanctioned the study. Clinic managers where the study was undertaken also granted permission. Consent was also obtained from the participant nurses, parents and care givers. Codes rather than actual identities of the participants were used to gather and record the data. Participants were also dully informed of their right to withdraw from the study at any time of the process should they found it fit to do so. Participants were assured that their withdrawals would not affect their democratic and legal right to receive any service from the clinics concerned.

The specific objectives of this paper were to:

SO2: investigate the socio-demographic characteristics of the observed nurses.

SO3: observe the frequency of nurses who would record the personal information of the child on the RtHC.

SO4: observe the recordings performed by nurses on the RtHC focusing on, for example, growth monitoring instrument; weight and height, immunization, nutritional statuses of the children and Vitamin $\mathrm{A}$ recording.

SO5: investigate how the nurses gave feedback to the Care givers of the children.

SO6: determine the quality of the RtHC in the Makhado municipality clinics.

In pursuit of achieving these objectives, the discourse of this paper was guided by the following research questions:

RQ1: What is the administrative and managerial pattern adopted by nurses on the RtHC among clinics in the Makhado municipality? This question is necessitated by the desire to probe issues raised by the main objective of this paper (SO1).

$\mathrm{RQ2}$ : What are the socio-demographic characteristics of the observed nurses for this paper? There is a need to investigate any links to the performance of the nurses on the administration and management of the RtHC. This question is intended to guide $\mathrm{SO} 2$ of this paper.

RQ3: What is the frequency of nurses who would record the personal information of the child and that of the mother on the RtHC? The premise is that if this record is not known, the instrument therefore means nothing. This responds to $\mathrm{SO} 3$ of this paper.

RQ4: How did the nurses perform recordings on the RtHC with regard growth monitoring instrument; weight and height, immunization, nutritional statuses of the children and Vitamin A recording? These are critical 
areas of the RtHC and if not recorded or properly recorded, this might compromise the quality of the RtHC, which might have some serious consequences. This question directs SO4 of this paper.

RQ5: How did the nurses give feedback to the care givers of the children visiting the clinics for the RtHC service? This question directs SO5 of this paper.

RQ6: What is the quality of the RtHC in the Makhado municipality clinics after experiencing so many complexity factors in its administration and management? This question addresses $\mathrm{SO} 6$ of this paper.

Looking at these objectives and research questions, it is evident that this paper goes beyond the scope covered by Kitenge \& Govender (2013) in their paper also conducted in the Makhado municipality on the management processes of the RtHC. Kitenge \& Govender (2013) in the main reported on professional nurses' challenges in the management of the RtHC, in addition using an urban-based facility and only focusing on establishing the professional nurses' difficulties on the administration of the RtHC. The Kitenge \& Govender (2013) paper present an urban environment while the current paper has a rural environment. Materially, this contrast is expected to bear different outputs. Furthermore, methodological application by Kitenge \& Govender (2013) which would only be questionnaire-based, and only administered on professional nurses only bring totally different approaches to the two studies as the current paper goes on a multi-disciplinary methodological approach. Kitenge \& Govender (2013) concern themselves with the personnel and the challenges they experienced in the administration of the RtHC while the current study concerns itself with the RtHC instrument however observing how the various categories of nurses administered the instrument. These alone would make considerable contrast in the outcomes of the current study and that of Kitenge \& Govender (2013).

\section{Literature Review}

A sizeable number of literature in the health sciences (Chersich et al., 2016; Hamad et al., 2016; Kitenge \& Gevender, 2013; Nyarko, 2014; Tarwa \& de Villiers, 2014; Mugo et al., 2015; Mulaudzi, 2012; Win, 2015) present child health as one of the most crucial inquiries of modern health sciences - especially in low and middle-income regions the world over. Win (2016) argues that poor growth monitoring of children has been a serious health hazard threatening millions to lives among children all over the world. Win (2016) furthermore reveals that this has been "a major source of early death for children under five [years of age] worldwide". This complexity could provide some serious challenge to global socio-economic development agenda - especially for low and middle-income regions because the impact of this factor on poverty reduction, economic development, national productivity and societal stability attempts and targets could be seriously impeded (Mugo et al., 2015).

Daniel et al. (2017) also added a voice contending that "a process of following and assessing the growth rate of a child in comparison to a standard by, periodic, frequent anthropometric measurements" be globally adopted to prevent associational consequences to these complexities. Compromising child health puts to challenge the imperatives expressed by the United Nations (UN) rhetoric "Every Woman, Every Child" (Mugo et al., 2015) for quality health care access. The UN rhetoric "Every Woman, Every Child" (Mugo et al., 2015) was meant to lobby for and mobilise financing, strengthen policy, and improve global service delivery in health care services (Mugo et al., 2015). Child health is a non-negotiable imperative of global health and development agenda promoted among others by the imperatives of the Millennium Development Goals (MDGs) as expressed in MDG4, MDG5 and MDG6 specifications. These Millennium Development Goals (MDGs) are now called Sustainable Development Goals (SDG) (Mugo et al., 2015; UN 2015). Furthermore, other access to health promotion initiatives were encoded in the Alma-Ata Declaration "health for all" rhetoric targeting advocacy for promotion of human welfare and better quality of life among the signatory regions of this declaration (Dhai, 2011).

The Alma-Ata Declarations postulate that the success of the "health for all" rhetoric would also significantly contribute to better quality of life, global peace and security around the world therefore ensuring and guaranteeing "sustained economic and social development" in global economies (Dhai, 2011). It is crucial in view of these global imperatives that economies around the world prioritised growth monitoring among children - especially those under five years of age to ensure emergence of healthy nations. Growth 
monitoring of children under five years of age should therefore be adopted a global practice to avoid and/or eradicate the associational socio-economic consequences. Global health scholars such as Chersich et al. (2016) argue that child health should be safeguarded and promoted at the first identification of the pregnancy of the mother right through the early stages of child development. Proponents of this assertion argue that pregnant mothers should instead receive assistance as early as conception. These proponents contend that providing support to pregnant mothers the earliest would ensure proper health and development of the child and this might reduce resultant lifelong consequences associated with unmonitored and unsupported health care (Chersich et al., 2016). Future health complexities affecting humans are often associated with the past; how issues of health were handled at some early stage of life might have bearing on future adult health for example (Hamad et al., 2016). In support of this assertion, Mulaudzi (2012) contended thus "We worry about what a child will become tomorrow, yet we forget that he is someone today" having borrowed this reference from Stacia Tauscher. This paper studies child health by observing how the Road to Health Chart (RtHC) as an instrument monitoring child health is administored in rural clinics of South Africa. The RtHC is currently referred to as the Road to Health Booklet (RtHB) in some modern health science literature following modifications on the instrument (Mulaudzi, 2012). It is imperative to note that the older RtHC was an A3 chart whereas the modern RtHB is a B5 booklet which uses different colours between boy and girl children (Mulaudzi, 2012). Since the clinics where this paper was conducted were using the RtHC during the study process, this paper refers to this instrument as the RtHC instead.

The RtHC could be described as an instrument containing what Hamad et al. (2016) would call "early life determinants of health". These determinants of health would influence health outcomes in future - not only for an individual but the entire nation (Hamad et al., 2016). These consequences might even move beyond local and regional political paradigms stretching into the entire global network. It could be that any threat to provision of health from an individual point of view to the local and regional paradigms - especially at early life of the population could become significant compromise of attainment of global quality health global standards as determined through efficient health access and provision by all as postulated by both the United Nations (UN) and the World Health Organisation (WHO) for instance.

The UN and the WHO have been on a global road show to lobby countries and campaign for "a human rights-based approach to health" (Bustreo et al., 2013) because these organisations believed that health is on its own an incontestable human right (London, 2008). Resultantly, and in collaboration of this assertion, London (2008) argued that "health as a right has been extensively elaborated and needs no repeating". The human rights-based approach to health "aims to realize the right to the highest attainable standard of health (or "right to health") and other health-related rights" (Bustreo et al., 2013). In view of this global approach on ensuring and improving quality global health of humans, it is critical for everyone charged with the responsibility of dispensing health service to understand that health is a basic human right, and should be treated as such. The post-apartheid South African constitution demonstrates this imperative by having listed access to health for its citizenry as a basic human right factor. This constitutional mandate is enshrined in the constitution of the republic in Chapter 2 of the Bill of Rights. The Constitution of the Republic of South Africa, Act 106 of 1996 says in section 27 "everyone has the right to have access to health care services" (Dhai, 2011). Evidently and effectively, the post-apartheid South African state postulates that each citizen should be aware of this right, in as much as everyone who is charged with the responsibility of dispensing public health service to the citizenry should.

Every public health worker has a responsibility to ensure that this human right is accordingly realised (London, 2008). This basic human right is also guaranteed for access by all through public policy blueprint which the post-apartheid state developed. For example, the post-apartheid South African government in 2004 promulgated "the National Health Act 3 to provide a framework for a structured and uniform health system that took into account the obligations imposed by the constitution" of the republic (Dhai, 2011). In addition, the South African post-apartheid government developed the White Paper on Transforming Public Service Delivery which came to be known as Batho Pele White Paper of 1997 (Notice No. 1459 of 1997) (Cloete, 2002). The main objectives of this document was to facilitate for a public service system which would ensure that public service was more efficient, effective and economical when dispensed to the 
citizenry at large (Cloete, 2002). According to Cloete (2002), Batho Pele means "People First", and this was founded on the post-1994 public service motto of "Service to the People" which sought to promote prioritisation of quality service provision to the South African public as driven by the constitutional mandate enshrined in the constitution of the Republic. Any form of violation of this fundamental human right; intended or unintended could be unconstitutional, and that violation might also be evidence of maladministration for public servants who fail to comply with public expectations of it. Some scholars of constitutional human rights and health such as London (2011) argue that there is that need for governments to develop and provide relevant personnel training and development in the health sector on issues of human rights and its subsequent relationship to health access and promotion for example.

The training could ensure adherence to this constitutional obligation by the state and public health workers in particular. London (2011) cites the Health and Human Rights Programme in the School of Public Health and Family Medicine at the University of Cape Town (UCT) as a typical skills development short course offered to public health practitioners and service providers to 'Train-the-Trainer' in health and human rights for example. Bustreo et al. (2013) reveal that the right to health might include among others timely and appropriate health care as well as the underlying "determinants of health". Globally, child health is a human right which is highly prioritised by a plethora of organisations and stakeholders to human right and health promotion such as the UN and the WHO. In fact, Mugo et al. (2015) went on to argue that maternal, newborn and child health $(\mathrm{MNCH})$ were serious contenders to the priority list of international communities in different regions of the world, and these have received substantial attention on their health and development agendas. It is recommended by organisations such as the UN and the WHO that growth of the child be continuously and consistently monitored. The monitoring might have to begin from birth until such time the child reacheed at least five years of age (Win, 2016). This growth monitoring by health authorities of respective countries should be conducted monthly and/or bimonthly at the least. The recordings should be plotted on the child's RtHC.

The recorded information on the child should be interpreted and communicated among health workers and caregivers of the respective child from as early as birth until the child reached at least five years of age (Bilal et al., 2014). Despite these clear and unambiguous standards and provisions, the RtHC remains the most ignored instrument in most public health institutions and service points the world over. For instance, Mulaudzi (2012) found that the majority of health care workers in South African health care centres failed to record the information on the RtHC. On the other hand, approximately $54 \%$ a the doctors would ask for the child's RtHC from care givers while a few doctors $(7 \%)$ would record the child patient's information on the RTHC when visiting health facilities (Mulaudzi, 2012). Evidently, the RtHC is mismanaged and improperly administered in public health institutions in South Africa. Senoge (2011) and Win (2016) also demonstrate this shortcoming in the South African health system in the Ethekwini Metropolitan area and the West Rand rural clinics respectively. Based on this challenge as it relates to South Africa, the purpose of this paper is to provide a corrective and preventive blueprint which would suggest ways and means to address the challenge. In doing so, this paper in the main investigates the administrative and managerial patterns on the RtHCs in Makhado municipality clinics (SO1).

\section{Research and Methodology}

\section{Study design}

This is a quantitative-qualitative study. This section describes the procedure followed in conducting the study; that is the ethical considerations of the study, the nature of the study, the sampling method and procedures, the method and instruments used in the collection, analysis and interpretation of data, and finally the theoretical underpinnings informing the analysis of the study. The results of this paper are thereafter reported, conclusions and policy recommendaions presented.

\section{Problem statement and theoretical underpinnings}

This paper is built on premise that evidence exists that the South African health system is characterised by serious omissions with regard the administration and management of the RtHC by public health workers, and in particular in state service facilities (Senoge, 2011; Mulaudzi, 2012; Win, 2016). Senoge (2011) found 
that level of knowledge and performance in the administration and management of the RtHC is unacceptably lower than expected considering that approximately $62 \%$ of public health workers struggled with the technical issues of the administration and mnagement of the RtHC instrument. This paper adopted its theoretical underpinnings from Win (2016) - especially with regard analysis of the complexities, barriers and bottlenecks affecting proper administration and management of the RtHC among the selected participant clinics. The variables guiding this paper were constructed from related studies (Senoge, 2011; Win, 2016) and departmental guidelines on the RtHC.

\section{Ethical considerations}

Ethical clearance was granted by the University of South Africa (UNISA) where the main study was academically registered and conducted from. The Limpopo Provincial Department of Health also sanctioned the study. Clinic managers where the study was undertaken also granted permission. Consent was also obtained from the participant nurses, parents and care givers. Codes rather than actual identities of the participants were used to gather and record the data. Participants were also dully informed of their right to withdraw from the study at any time of the process should they found it fit to do so. Participants were assured that their withdrawals would not affect their democratic and legal right to receive any service from the clinics concerned.

\section{Description of the study area}

The study was undertaken in the Makhado municipality of Vhembe District, Limpopo Province (figure 1).

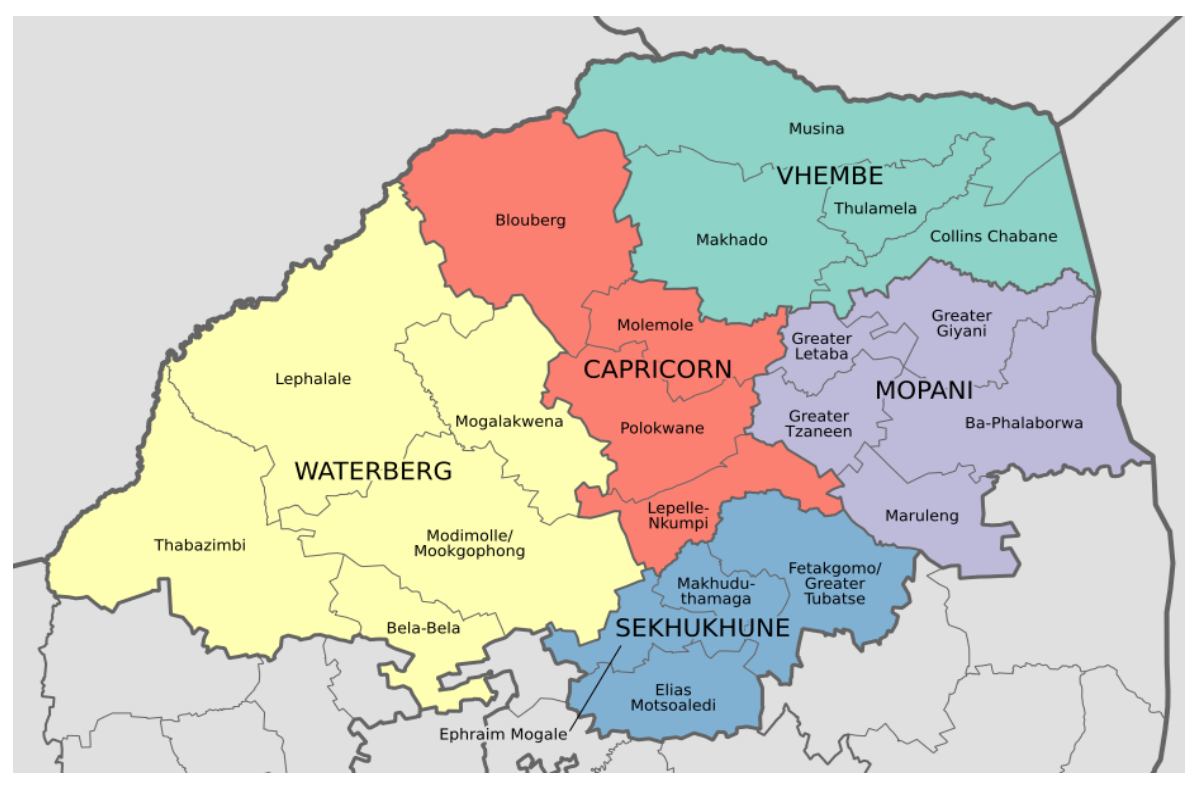

Figure 1: Map of the Limpopo province with the Vhembe District and Makhado municipality depicted

Limpopo Province is one of the nine provinces of the Republic of South Africa. The province is situated in the far north of the country bordering Zimbabwe in the north, Mozambique in the East, Botswana in the north West while the Gauteng Province of South Africa is in the south. Limpopo Province has approximately 5.6 million inhabitants. The province has five political and administrative districts; Vhembe, Mopani, Capricorn, Waterberg and Sekhukhune. Vhembe has an estimated population of approximately 1.2 million people; the majority being Tshivenda (76.3\%), XiTsonga (24.9\%), Northern Sotho (1.6\%), Afrikaans $(1.3 \%)$ and followed by the rest of other languages speakers. The Vhembe district municipality is made out of four local municipalities; Collins Chabane in the South East, Makhado in the South West, Musina in the North and Thulamela in the Centre. This paper concerns Makhado local municipality. The 
Makhado Local Municipality is a Category B municipality. The municipality borders with Musina in the north, Greater Giyani in the south (Mopani District), Thulamela in the east, and Molemole in the west (Capricorn District). The municipality covers a geographical space of approximately $8300 \mathrm{~km}^{2}$. Makhado local municipality was first established on 31 October 1934 as the apartheid era Louis Trichardt Town Council. Post-apartheid, new municipal demarcations were effected, and a number of areas including formerly black homeland areas were incorporated into this new municipality now known as the Makhado local municipality. Makhado municipality is divided into four regions: Makhado (previously Louis Trichardt), Vuwani, Dzanani and Waterval sub-areas.

\section{Sampling and sampling procedures}

Six participant clinics $(n=6)$ were randomly slected from a total of 12 clinics located in the Makhado municipality clinics area. The clinics were chosen based on accessibility by the researcher. The choice of these clinics took into account the long distances in between the respective clinics and the bad conditions of some of the dirt roads characterising the area. The inclusion criteria for the clinics were based on the respective clinics rendering comprehensive child care services to the respective communities each clinic was serving. Clinics in the study area are administratively divided into two local areas (1A \& 2B). Each of these local areas administers six clinics. For this paper, three $(n=3)$ clinics were randomly selected from each local area. A purposive convenience sampling of study subjects; nurses on duty, parents or caregivers who had brought their children to the clinic on the day of the study was conducted. Two nurses (one registered nurse and one enrolled nursing assistant) per clinic were randomly selected for observations. These participants were observed while rendering care - and when administering the RtHC to the children under five years of age. Enrolled nurses were included in the study because they are part of health care providers although their scope of practice might have limitations on what care they could provide. This paper used retrospective record review of the RtHCs in participant clinics. This method was successfully adopted by Win (2016). A total of 36 children's RtHCs were reviewed during the process. This exercise was followed by scanning of the socio-demographic information of the participant nurses. The socio-demographic data on the nurses included among others the educational achievements, experience as a nurse, short course trainings undertaken and special training acquired.

\section{Method and instrument}

This paper followed a multi-method approach in its discourse. Observation, document review and personal interviews were adopted. Selected nurses were observed while administering and managing the RtHCs. Some selected RtHCs were also reviewed. Open-ended questionnaire-based instrument was used to conduct face-to-face interviews on participant nurses. The questionnaire instrument was in the local native Tshivenda language having considered that participants needed to be fluent when responding to the questionnaires. English is a problematic language despite the fact that all schools teach the language. Mafukata (2012) provided the guidelines for construction and development of a cross-language questionnaire. The guidelines are as indicated in table 1.

Table 1: Guidelines for translating the English to Tshivenda questionnaire

\begin{tabular}{|l|l|}
\hline Guideline & Objective \\
\hline Pragmatic translation & $\begin{array}{l}\text { Ensures that the content of the English questionnaire is accurately } \\
\text { communicated in the target language of the respondents (Tshivenda) }\end{array}$ \\
\hline Aesthetic-poetic translation & $\begin{array}{l}\text { Ensures that the moods and feelings of the source language used in the } \\
\text { original questionnaire are maintained in the target language }\end{array}$ \\
\hline Ethnographic translation & $\begin{array}{l}\text { Ensures that meaning and cultural content of the translated local dialect } \\
\text { questionnaire matched the expectations of the respondents }\end{array}$ \\
\hline Linguistic translation & $\begin{array}{l}\text { Ensures that the grammatical forms and equivalent meanings in } \\
\text { Tshivenda were correctly presented }\end{array}$ \\
\hline
\end{tabular}

Adapted from: Mafukata (2014) 
In a follow-up study, Mafukata (2014), reasoned that face-to-face interviews conducted in foreign languages such as English among third language speakers such as the participants in this current paper often led to distortion of responses. This translation is in agreement with this assertion. Finally, structured exit interviews were also conducted on the care givers of the children to control and validate the findings. A check list was used to guide the processes of the data obtained through observations.

\section{Results and discussion}

Two approaches were used in data analysis. First to be analysed was the quantitative data. SPSS version 23 was used to analyse this data. Simple descriptive analysis was performed and the results recorded in clear figures. The qualitative data were thematically analyzed.

This section presents the results of this paper. First, this paper presents results on the administrative and management competence of the nurses with regard the RtHC. Secondly, the result regarding the demographical characterisation of the nurses are presented. This logic followed critical assumptions that the performance of the nurses on the administration and management of the RtHC would hinge on the nurses' socio-demographic characteristics.

\section{Administrative patterns adopted by nurses on the RtHC}

This section presents the results on the administrative and management patterns adopted by the nurses on the RtHC in the Makhado municipality clinics. The results are reported on the variables randomly selected from the child's RtHC for the purpose of this paper. The objective of this section is to pinpoint how the nurses administered and managed the RtHC in the selected clinics. Although the results of this paper revealed that there were incidences where information was dully recorded, there were however some considerable omissions identified; either through unrecorded or incorrectly recorded data on the RtHC. The results of this paper (figure 2) revealed that recordings on the child's personal information was in the majority recorded $(90 \%)$ while in some incidences $(10 \%)$ that information was not recorded.

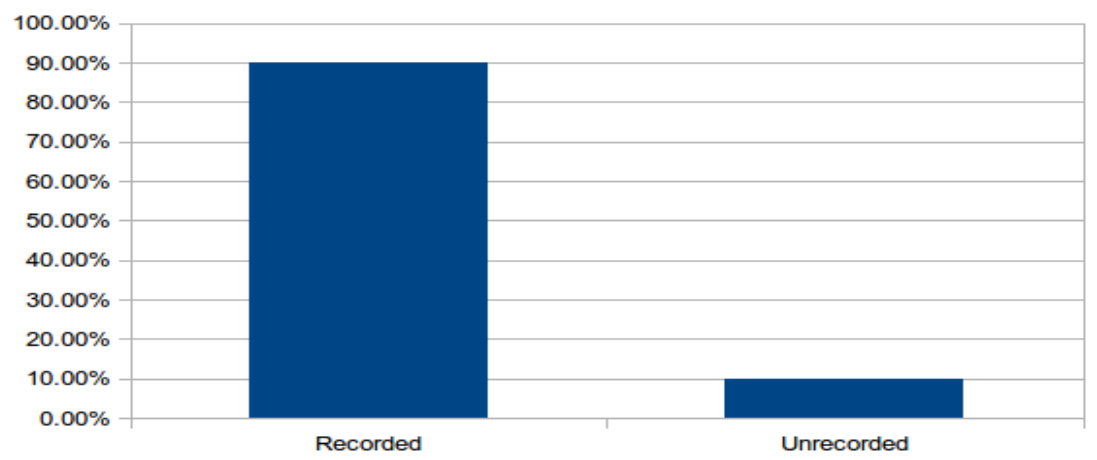

Figure 2: Child's personal information recording on the RtH

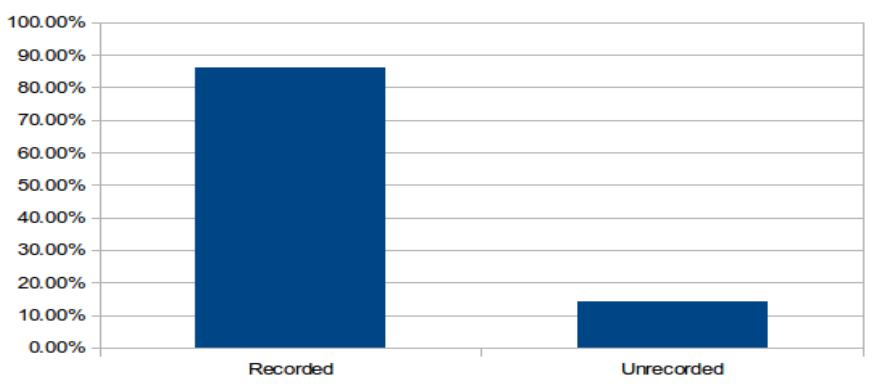

Figure 3: Mother's personal information recording on the RtHC 
The results of this paper also revealed that the mother's personal information was recorded in $86 \%$ of the RtHCs while $14 \%$ of the RtHCs contained unrecorded mother's personal information (figure 3). This result could not mean much with regard monitoring the child's health because variables were observed randomly. Serious complexities were identified when variables were measured in singles. As reflected in figure 4, the majority of the RtHCs identified who the owners of those instruments were because the names of the children were in most cases recorded on the instruments. This result suggests that the recorded information could be used to address complexities affecting real owners of the instruments because the owners were known. On the individual variables such as the name of the child being a variable for personal identification of the child and the address of the child, the results were as illustrated in figure 4.

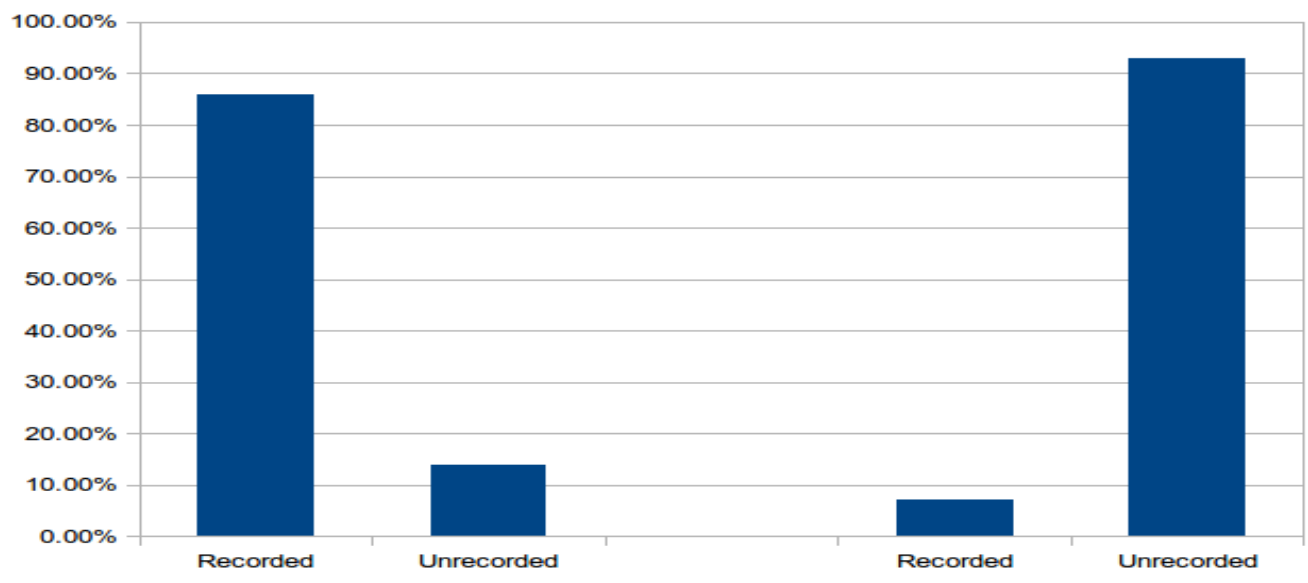

Figure 4: Selected variables on the child's information recordings on the RtHC

Although indications were that the personal information of the child as measured by the names and the address of residence of the child on the RtHC, the results revealed that the names of the children were in the majority unrecorded $(86 \%)$ with only $14 \%$ recorded. The results revealed that the child's residential address was in the majority unrecorded $(92.9 \%)$ with a mere $7.1 \%$ of recordings. This paper probed this differences in the recordings considering that these recordings were conducted by the same nurses, on the same instrument and on the same day. The question was "what might be informing this complexity?" The results revealed that although the recording on the child's names was considerably higher on the RtHCs, the names of the children were in the majority entered by the care givers, and not the nurses. Care givers would be instructed to enter the names of the children on the RtHCs. Those missing could be those who are illiterate, or who simply failed to comply. Elsewhere in the paper, it has been revealed that even in instances where information was recorded in the majority, in the same way as in the names of the child for example, such information would be in the main incorrectly recorded. The implication of this on growth monitoring of child health are also dully illustrated there.

The critical question to ask was therefore "what are the factors informing this pattern in the clinics" Earlier studies such as Win (2016) for example have suggested that operational factors in the clinics were the main determinants of the resultant patterns on the RtHC administration and management. This paper however found that what Win (2016) found in the Western area of Gauteng Province regarding nurses having challenges on the administration and management of the RtHC as being informed by operational factors could not be established in this study area. Secondly, level of education, skill development and work experience with regard exposure to the RtHC of the nurses who administer the RtHCs have been cited as the factors influencing this poor trend on the RtHC in clinics in South Africa. To test if educational levels and experience might have been playing any role in the state of affairs in the selected clinics, performance of the nurses on the administration of the RtHC between registered and enrolled nurses were conducted. The paper measured how each group administered and managed the RtHC on some specifically selected variables. Table 2 displays the results. 
Mudau and Human / International Journal of Research in Business and Social Science,

Vol 6 No 3, 2017 ISSN: 2147-4486

Table 2: Nurses' administration and management of the RtHC on some selected variables

\begin{tabular}{|c|c|c|c|c|}
\hline \multicolumn{3}{|l|}{ Registered nurses } & \multicolumn{2}{|l|}{ Enrolled nurses } \\
\hline Variable & Recorded & Unrecorded & Recorded & Unrecorded \\
\hline Breast feeding & $7.1 \%$ & $92.9 \%$ & $8.1 \%$ & $81.8 \%$ \\
\hline Head Circumference & $21.4 \%$ & $78.6 \%$ & $8.1 \%$ & $81.8 \%$ \\
\hline Height & $4.3 \%$ & $85.7 \%$ & $9.1 \%$ & $90.9 \%$ \\
\hline
\end{tabular}

Table 2 shows that only a handful of nurses; registered nurses $(7.1 \%)$ and enrolled nurses $(8.1 \%)$ recorded information on Breast feeding on the RtHCs. This result suggests that the larger majorities of both enrolled and registered nurses incorrectly administered the RtHC at their clinics. Suggestively, information on Breast feeding was therefore missing, and this could be problematic with regard growth monitoring of the respective children whose information could not be recorded. Missing information on the RtHC has been problematic for some health systems as evidenced by the findings reported by Schoeman et al. (2006). Schoeman et al. (2006) contended that missing information would put the health of the child at risk. It is difficult for health providers to effect efficient intervention measures on the children to monitor their growth as expected and required because crucial information of their health would be missing. Furthermore, the results of this paper revealed that a mere $21.4 \%$ of registered nurses versus $81 . \%$ of enrolled nurses recorded information of the head circumference on the RtHC whereas recordings were poorest among registered nurses on the height $(4.3 \%)$ while the recordings of the enrolled nurses being slightly higher than those of the registered nurses at $9.1 \%$. Even though the recordings of the registered nurses were a bit higher, both recordings remain extremely poor. Evidently, the results of this paper suggest that qualifications and experience of the nurses might not be the determinant factors of the poor administration and management of the RtHC at these clinics. The results revealed in some instances; that is, areas of measurement as indicated in table 2 that the lesser qualified and experienced enrolled nurses performed much better than their better qualified and experienced registered nurses counterparts. This paper also measured the performance of the registered nurses on the administration and management of the RtHC on certain areas of the RtHC (Serelogy - 1\&2; gestational age - 3\&4; nutritional assessment $-5 \& 6$ and immunisation - 7\&8) without comparing them with the enrolled nurses. Each variable was measured on whether it was recorded or unrecorded. The main aim of this investigation was to determine the quality of the instrument. It is good that the children and the care givers kept the RtHC but if that instrument's quality is compromised, then the instrument is rendered useless and unhelpful with regard growth monitoring of child health.

In addition, the results of this paper revealed that the mother's personal information on serology (1 \&2) for example was not recorded in at least $92.9 \%$ of the RtHC. This result suggests that only $7.1 \%$ of mothers had their serology record on the child's RtHC recorded. The results furthermore revealed that information on the gestational age on delivery ( $3 \& 4)$ were absent on $94.4 \%$ RtHCs while information on nutritional assessment $(5 \& 6)$ was better recorded with that of immunisation $(7 \& 8)$ however largely unrecorded.

The results of this paper had to inform preventive and/or corrective measures with regard the evident poor performance of nurses of all categories in the administration and management of the RtHC in the Makhado municipality clinics. However, the preventive and/or corrective measures could best be determined if complexity factors influencing this outcome could be correctly identified. Assumptions made by this paper were that the results of this paper as they are reflected would not be able to lead such intervention. The results only reflect the state of performance on the administration and management of the RtHC without forwarding the causality factors which would be the complexities. This was an interesting point to make follow up on for the purpose of the paper. The researcher determined to randomly and personally interview 
some enrolled and registered nurses to establish the complexity factors which might be playing influence to this critical outcome. The results revealed some intertwined factors. Indications were that, although the majority of enrolled nurses would reveal that they lacked sufficient expertise on the RtHC administration and management, the majority of the nurses however revealed that nurses simply would not take the RtHC as a child health tool as critically as a trained nurse was supposed and expected to do. This outcome is corroborated in the responses of the nurses elsewhere in this report who revealed that there was no longer any motivation and pride for a nurse to practice as a nurse. This determination was eroded by various factors. Some nurses argued that they were expected to work for long hours and consulting too many patients on a particular day, and they often would get tired before the end of business day. This finding of nurses having to work long hours is similar to that of Kitenge \& Govender (2013). Kitenge \& Govender (2013) reported that some nurses thought there was personnel shortages in the health facilities in the Makhado municiplity health facilities to an extent of causing nurses having to do too much work, and for long hours. It is unfortunate that this factor leads to some sloppy work - especially on issues such as the RtHC - unless there is evidence that the particular child is sick to warrant any special attention. A condideable number of resondent nurses conceded however that some lazy nurses would capitalise on this factor to do slopy work for mere routine. Clinic managers' lack of supervisory work of the nurses promote leizures fare among the nurses. Nurses lacked pressure from the care givers who, because of illiteracy would not probe for answers were they would be questions to raise regarding the health of their children.

In addition, a follow up observation was made to establish if the recorded information was accurate or inaccurate between the registered and enrolled nurses (table 3 ).

Table 3: Measurement of the quality of the RtHC at Makhado municipality clinics

\begin{tabular}{|c|c|c|c|c|}
\hline \multicolumn{3}{|l|}{ Registered nurses } & \multicolumn{2}{|c|}{ Enrolled nurses } \\
\hline Status of record & Accurate & Inaccurate & Accurate & Inaccurate \\
\hline Head Circumference & $78.6 \%$ & $21.4 \%$ & $81.8 \%$ & $9.1 \%$ \\
\hline Weight of the child & $75.7 \%$ & $14.3 \%$ & $72.7 \%$ & $27.7 \%$ \\
\hline
\end{tabular}

These results were determined to measure the quality of the RtHC at these clinics. The results revealed that there were incidences of inaccurately recorded information on the RtHCs of the children by both the registered $(21.4 \%)$ and enrolled nurses $(9.1 \%)$ with regard the head circumference information of the child. Accuracy of information was reasonably higher with enrolled nurses $(81.8 \%)$ than with registered nurses $(78.6 \%)$. In addition, the results revealed that the weight of the child was accurately recorded by registered nurses $(75.7 \%)$ and enrolled nurses $(72.7 \%)$ while inaccurately recorded by both registered nurses $(14.3 \%)$ and enrolled nurses $(27.7 \%)$ respectively. That the inaccuracy of information was highest among registered nurses than among enrolled nurses with regard the head circumference of the child on the RtHC might point to some organisational deficiencies among the health personnel in these clinics. The gap of inaccuracies between the two groups is even too large. It might be that registered nurses took their job lightly than the enrolled nurses who might have had better attitude in this regard. However the factors need to be measured and identified. This has been addressed elsewhere in this report. Furthermore, it is critical to note that similar patterns on the RtHC were also identified elsewhere in the country; for example, among Gauteng provincial clinics (Kitenge \& Govender, 2013) and in some parts of Limpopo Province (Mothiba \& Tladi, 2015) this patterns have previously been reported. Suggestively, inaccuracy of information on the RtHC in the Limpopo provincial health jurisdiction seems to be rife. This might have some dire consequences on the quality of health services to the populace in the province. It is therefore critical that the issue of a possible compromise on the quality of the RtHC in the Limpopo provincial health system be further investigated. In fact, this paper hypothesised that studies on the quality of the RtHC instrument in the Limpopo province in particular were scares and rare. Based on this sentiment, this paper provides a 
snap shot investigation to back up its call for further probe on this issue in Limpopo Province in general. Although this paper was empirically limited to provide a full scope of such type of study, the present study still determined that it was necessary to reflect on the same. The results of this paper revealed that the quality of a substantial number of the RtHCs could have been seriously compromised. For instance, although recordings were made on the RtHCs from time to time, table 2 for instance revealed that a substantial number of some recordings was found to be inaccurate. Inaccurate information might be more dangerous than ungiven information. When incorrect recording is supplied to care givers and health workers, that information could mislead intervention measures therefore compromising child health imperatives while putting the child at physical risk on the one hand. It is lamentable and regrettable that the consequences of this could also negatively impact on the overall national health system. Supplying incorrect RtHC information practices might also lead to incorrect diagnoses of serious physical problems like stunted growth and under-nutrition among children which may lead to poor mental development, poor health and ultimately increased child mortality (UNICEF, 2016; UNICEF 2015; Bassett \& Ruel-Bergeron, 2014). Inconsistent recording and plotting of weight on the graph might lead to incorrect interpretation of growth pattern by both health workers and parents or the care givers frustrating the entire process of child growth monitoring and guarantee of quality of health dispensed to the public in general.

This paper postulates that the conduct informing administration and management of the RtHC among the nurses in the Makhado municipality clinics might be informed by the socio-demographic characteristics of the nurses in the clinics. In view of this, this paper delves into this critical area in its discourse. The results are as presented and discussed below:

The socio-demographic characteristics of the nurses

The results on the socio-demographic characteristics of the participant nurses are as reflected (table 4).

Table 4: Socio-demographic characteristics of the nurses in participant clinics

\section{Categories of the nurses}

$\begin{array}{ll}\text { Registered nurses } & : 39 \% \\ \text { Other nurses } & : 61 \%\end{array}$

\section{Experience in Primary Health Care}

$\begin{array}{ll}\text { Less than } 10 \text { years } & : 29.6 \% \\ \text { More than } 10 \text { Years } & : 71.4 \%\end{array}$

\section{Additional qualifications}

Community Nursing : $\quad(64.3 \%)$

PHC speciality and Midwifery: (42.9\%)

Short Course on-the-job training: Integrated Management of Childhood IIIness (IMCI): (71.4\%)

Prevention of Mother to Child Transmission of HIV (PMTCT) (64.3\%).

Treatment and management of Tuberculosis (TB), Human Immunodeficiency Virus and Acquired Immunodeficiency Syndrome (HIVIAIDS) (78.6\%)

Breastfeeding enhancement (64.3\%)

As the results of this paper reveal in table 4, thirty nine percent (39\%) of the nurses were registered nurses. All the registered nurses had more than 10 years of nursing experience. These results suggest that a sizeable number of the nurses had been exposed to the RtHC experience for a reasonably long period. This assertion could be corroborated from the experience of service in Primary Health Care (PHC) among the majority of the nurses. The results of this paper revealed that $71.4 \%$ of the nurses had long experience spanning over ten years in Primary Health Care (PHC) environment. The experience is sufficient enough 
for these nurses to know and understand the criticality and importance of the RtHC, the need for its proper administration and management, the resultant consequences of its poor administration and management among others. The nurses are also, as a result of their level of training; that they were registered nurses with long experience in child health expected to have sufficiently mastered the technical abilities to properly administer and manage the RtHC. With advanced qualification levels as displayed in the results of this paper, it would be expected that there were minimal challenges on the administration and management of the RtHC among the nurses in these clinics. For example, the results of this paper revealed that the majority of registered nurses $(64.3 \%)$ had additional qualifications in Community Nursing, and PHC speciality. A considerable other (42.9\%) held additional qualifications in areas such as midwifery $(42.9 \%)$. In addition, the department had also conducted skills courses through on-the-job training as short courses such as Integrated Management of Childhood Illness (IMCI) on $71.4 \%$ of the nurses while $64.3 \%$ of the nurses had received further skills training and development in Prevention of Mother to Child Transmission of HIV (PMTCT), Treatment and management of Tuberculosis (TB), Human Immunodeficiency Virus and Acquired Immunodeficiency Syndrome (HIVIAIDS) (78.6\%) and Breastfeeding options (64.3\%) for example. The qualifications reveal a highly qualified workforce with considerably high job experience. Under normal circumstances, it would be expected that challenges experienced by nurses in these clinics would in all probability be lower than what the results of this paper found.

However, the omissions displayed by some of these nurses in the administration and management of the RtHC might point to the need to investigate the factors influencing the difficulties experienced by the nurses in these clinics. This analysis of the factors was carried based on the guidelines provided by Win (2016). Win (2016) found that the complexities experienced by nurses on the administration and management of the RtHC might be emanating from the fact that not all the nurses were exposed to the RtHC on daily basis. Win (2016) goes on to reveal that there are in fact nurses who were occasionally and/or frequently exposed to the administration and management of the RtHC. The exposure of these nurses to the RtHC is determined by operational factors among others. For instance, some nurses would only be required to deal with adult patients, and only deal with child patients when those nurses assigned to such responsibilities were incapacitated to render such service for various reasons (Win, 2016). The assertion here is that not all the nurses were exposed to the RtHC all the time. The poor performance of the nurses on the administration and management of the RtHC therefore necessitates an investigation to establish the reasons for such performances. In this regard, the questions to be asked might have to do with whether these nurses were not properly exposed to the RtHC administration and management through daily practice, or sufficiently trained with regard issues of the RtHC, or whether these nurses were merely incompetent and inefficient, or whether these nurses were lacking professional assistance by their health constituencies, and so forth. What makes the omissions even more puzzling and difficult to understand is that the RtHC has been found by most health workers and nurses who administer and manage it often as being a good instrument which was easy to understand, administer and manage because the document would provide "a clear scope with comprehensive guidance for the nurses" with regard its administration and management (Win, 2016).

\section{Conclusion}

The main objective of this paper was to investigate the administrative and managerial patterns employed by nurses on the RtHC among randomly selected clinics in the Makhado municipality. Retrospective observations of the processes of recordings the nurses performed on the RtHC ( $\underline{n}=36)$ were carried out to collect data. A checklist was used to aid the observations. Face-to-face interviews were also administered on the nurses in collaboration with document review. The results of this paper revealed considerable omissions on the administration and management of the RtHC among the selected clinics. Some crucial information was also incorrectly recorded while some was never recorded at all. The majority of nurses also failed to provide feedback to the care givers of the children after conducting the RtHC processeses. It is recommended that clinic managers develop monitoring instrument(s) for nurses dealing with RtHC administration and management in order to enforce compliance with the requirements of a quality RtHC. Regular in-service training, refresher seminars and workshops for the nurses on how to administer and 
manage the RtHC should be conducted. Care givers should receive timeous feed back on the growth monitoring processes of their children.

\section{References}

Bilal, S.M., Moser, A., Blanco, R., Spigt, M. \& Dinant, G.J. (2014). Practices and challenges of growth monitoring and promotion in Ethiopia: A Qualitative study. Journal of Health Population and Nutrition, 32 (3): 441-451.

Bassett, L, and Ruel-Bergeron, J. (2012). Promoting Healthy Child Growth and Development: advances and opportunities for community-based nutrition nrograms in Central America. World Bank.

Bustreo, F., Hunt, P., Gruskin, S., Eide, A., McGoey,L. Rao, S., Songane, F., Tarantola, D., Unnithan, M., Yamin, A.E., van Bolhuis, A., Ferguson, L., Halliday, E., Kuruvilla, S., Popay, J. \& Genevieve, S.G. (2013). Women's and Children's Health: Evidence of Impact of Human Rights. World Health Organization, Geneva, Switzerland.

CDC. (2013). Use and interpretation of the WHO and CDC growth charts for children from birth to 20 years in the United States. National center for chronic disease prevention and health promotion. Retrieved from: https://www.cdc.gov/nccdphp/dnpa/growthcharts/resources/growthchart.pdf. Accessed [26 September 2017].

CG2, (2010). Record Keeping Guidelines. Clinical Governance. Volume 3 March 2010.

Chersich,M.F., Luchters, Blaauw, D., Scorgie, Kern, E., van den Heever, A., Rees, H., Peach, E. \& Kharadi, S. (2016). Safeguarding maternal and child health in South Africa by starting the Child Support Grant before birth: Design lessons from pregnancy support programmes in 27 countries. SAMJ, 106(12): 1192-1210.

Cloete, M. (2002). Service delivery in the South African public service: Implementation of the Batho Pele principles by Statistics South Africa. Unpublished Masters dissertation, University of Pretoria, Pretoria.

Department of Health. (2014). Infant and young child feeding policy. Pretoria: Government Printers.

Department of Health. (2013). Mother, child health and nutrition booklet. Pretoria: Government Printers.

Daniel, B., Tesfaye, N., Mekonin, E., Kassa, A., Mensur, K., Zerihun, E., Deriba, K., Tadesse, H. \&Tomas , Y. (2017). Knowledge and Attitude on Growth Monitoring and its Associated Factors among Mothers/Guardians of Children Less than Two Years in Areka Town, Southern Ethiopia. Journal of Nutritional Disorders \& Therapy, 7(3): 216. DOI: 10.4172/2161-0509.1000216.

Dhai, A. (2011). Healthcare reform in South Africa: A step in the direction of social justice. South African Journal of Bioethics and Law, 4(2): 48-49. Global nutrition report. (2014). Nutrition Country Profile. Swaziland.

Hamad, R., David H. Rehkopf, D.H., Kuan, K.Y. \& Cullen, M.R. (2016). Predicting later life health status and mortality using state-level socio-economic characteristics in early life. Population Health, 2(2016): 269-276.

Joubert, K., \& Casoojee, A. (2013). Hearing-screening record-keeping practices at primary health care clinics in Gauteng. S Afr J CD, 60: 27-30.

Kitenge, G. \& Govender, I. (2013). Nurses' monitoring of the Road to Health Chart at primary healthcare level in Makhado, Limpopo Province. South African Family Practice, 55(3): 275-280, DOI: 10.1080/20786204.2013.10874350.

London, L. (2011). Training trainers in health and human rights. South African Journal of Bioethics and Law, 4(2): 106. 
London, L. (2008). What is a human rights-based approach to health and does it matter? Health and Human Rights, 10(1): 65-80.

Mafukata, M.A (2014). Undertaking Effective Cross-Language Questionnaire-Based Survey in Illiterate and Semi-Illiterate Rural Communities in the Developing Regions: Case of Communal Cattle Farmers in Vhembe District of Limpopo Province, South Africa. Journal of Arts and Humanities 3(1): 67-75.

Mafukata, M.A. (2012). Commercialisation of subsistance communal cattle production systems in the Musekwa Valley, Unpublished Ph.D thesis, University of the Free State, Bloemfontein.

Monyeki, M.A., Awotidebe, A., Strydom, G.L., Hans de Ridder, J., Mamabolo, R.L., \& Kemper, H.C.G. (2015). The Challenges of Underweight and Overweight in South African Children: Are We Winning or Losing the Battle? A Systematic Review. Int. J. Environ. Res. Public Health, (2015)12: 1156-1173.

Mothiba, T.M. \& Tladi, F.M. 2016. Challenges faced by professional nurses when implementing the Expanded Programme on Immunization at rural clinics in Capricorn District, Limpopo. African Journal of Primary Health Care \& Family Medicine, 8 (2): 1-5.

Mugo, N., Zwi, A.B., Botfield, J.R. \& Steiner, C. (2015). Maternal and Child Health in South Sudan: Priorities for the Post-2015 Agenda. SAGE Open, (April-June 2015): 1-14. DOI: $10.1177 / 2158244015581190$.

Mulaudzi, M. (2012). Road to health chart/booklet.Kalafong Hospital-University of Pretoria, Update 2012. Retrieved from: http://archivedpublicwebsite.up.ac.za/sitefiles/file/45/1335/877/UPdate\%202012Road\%20to_\%20Health.pdf. Accessed [28 September 2017].

Nyabuti, J.I. (2015). Factors associated with the continuation of growth monitoring among children 10 to 59 months old in Nyamira County, Kenya. Unpublished Masters dissertation, Kenyatta University, Nairobi, Kenya.

Nyarko, M.J. (2014). Management of the nutritional care of children under five years old by nurses in the Nelson Mandela bay health district. Unpublished Masters dissertation, University of South Africa, Pretoria.

Policy Brief. Child Nutrition Reauthorization (2015). Nourishing our children beyond the school day.

Schoeman, S.E., Hendricks, M.K., Hattingh, S.P., Benade, A.J.S. \& Dhansay, M.A. (2006). The targeting of nutritionally at-risk children attending a primary health care facility in the Western Cape Province of South Africa. Public Health Nutrition, 9(8): 1007-1012. DOI: 10.1017/PHN2006986.

Senoge, D. (2011). Exploring the practice of the health care workers regarding the use of the child growth and development monitoring tool (road to health card) in the Ethekwini Metropolitan area. Unpublished Masters dissertation, University of KwaZulu-Natal, Durban.

Statistics South Africa. (2014). Mortality and causes of death in South Africa, 2013: Findings from death notification / Statistics South Africa. Pretoria 2nd triennial report of the committee on morbidity and mortality in children under 5 years (COMMIC): November 2014.

Tarwa, C. \& de Villiers, F.P.R. (2007). The use of the Road to Health Card in monitoring child health. South African Family Practice, 49:(1):15-15d. Doi.org/10.1080/20786204.2007.10873497.

The Global Hidden Hunger Indices and Maps: An Advocacy Tool for Action. Retrieved from: http://www.VitaminsinMotion.com, Accessed [22 September 2016].

UNICEF. (2016a). From the first hour of life: Making the case for improved infant and young child feeding everywhere.

UNICEF. 2016b. One is too many: Ending child deaths from pneumonia and diarrhoea. 
Mudau and Human / International Journal of Research in Business and Social Science, Vol 6 No 3, 2017 ISSN: 2147-4486

UNICEF. (2014). Committing to child survival: A promise renewed. Progress report.

UNITED NATIONS. (2015). Sustainable development goals. Fact sheet. 